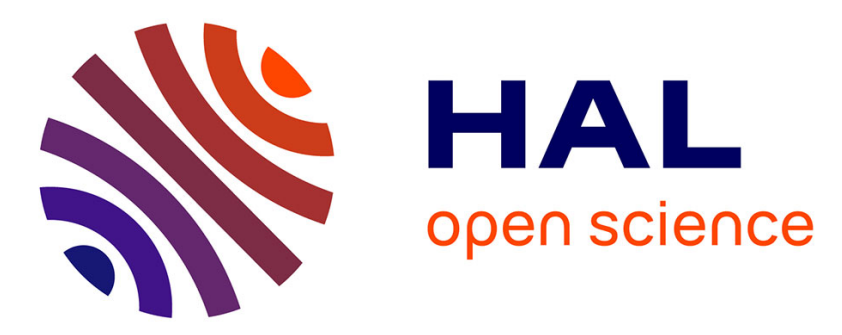

\title{
Experimental prediction of the vibration response of panels under a turbulent boundary layer excitation from sensitivity functions
}

Christophe Marchetto, Laurent Maxit, Olivier Robin, Alain Berry

\section{- To cite this version:}

Christophe Marchetto, Laurent Maxit, Olivier Robin, Alain Berry. Experimental prediction of the vibration response of panels under a turbulent boundary layer excitation from sensitivity functions. Journal of the Acoustical Society of America, 2018, 143 (5), pp.2954 - 2964. 10.1121/1.5037362 . hal-01922209

\author{
HAL Id: hal-01922209 \\ https://hal.science/hal-01922209
}

Submitted on 14 Nov 2018

HAL is a multi-disciplinary open access archive for the deposit and dissemination of scientific research documents, whether they are published or not. The documents may come from teaching and research institutions in France or abroad, or from public or private research centers.
L'archive ouverte pluridisciplinaire HAL, est destinée au dépôt et à la diffusion de documents scientifiques de niveau recherche, publiés ou non, émanant des établissements d'enseignement et de recherche français ou étrangers, des laboratoires publics ou privés. 


\title{
Experimental prediction of the vibration response of panels under a turbulent boundary layer excitation from sensitivity functions
}

\author{
Christophe Marchetto* and Laurent Maxit \\ Univ Lyon, INSA-Lyon, Laboratoire Vibrations Acoustique, F-69621 Villeurbanne, France \\ Olivier Robin and Alain Berry \\ Groupe d'Acoustique de l'Université de Sherbrooke, \\ Université de Sherbrooke, Sherbrooke, J1K 2R1, Canada
}

(Dated: April 4, 2018)

\begin{abstract}
This study aims at validating an experimental method for characterizing the vibration behavior of panels excited by a turbulent boundary layer excitation as a possible alternative to standard means like wind-tunnels or in situ tests. The approach takes advantage of an explicit separation of the excitation contribution from the dynamic behavior of the panel. Based on the measurement of deterministic transfer functions on the panel, called 'sensitivity functions', which are then combined with either measurements or a model of the wall-pressure fluctuations induced by the turbulent boundary layer excitation, the vibration response under such an excitation can be retrieved. For validation purposes, the wall-pressure fluctuations of the turbulent flow generated in an anechoic wind tunnel are measured with a flush-mounted microphone array. The decay rates and the convection velocity which mainly characterize the excitation are extracted from these measurements. The plate velocity response to this excitation is estimated following the proposed method using the measured sensitivity functions and the model of Mellen fed with experimentally estimated decay rates and convection velocity. A comparison between a directly measured vibration autospectrum under the actual flow and the one predicted following the suggested method shows satisfactory agreement.
\end{abstract}

PACS numbers: PACS: 43.40.At, 43.40.Dx

\footnotetext{
* christophe.marchetto@usherbrooke.ca
} 


\section{INTRODUCTION}

The experimental characterization of panels vibration under a turbulent boundary layer (TBL) excitation is of great interest for the transport industry and for researchers studying flow-induced vibration topics. Panel-like structures are mainly tested in wind tunnels or via in situ measurements. These experimental methods are hard to control, costly and subjected to variability between different laboratories and/or measuring techniques. Over the past decades, studies have been carried out to experimentally synthesize the pressure field induced by a TBL on a panel surface using an array of acoustic sources [1-3] and ultimately providing improved methods to characterize the vibration behavior of a panel under this excitation. However, these synthesis methods require a large number of sources (approximately 4 per smallest wavelength) to reproduce the small correlation lengths of the surface pressure field induced by the TBL, especially for subsonic velocities of the convected TBL. As frequency increases, the number of reproduction sources thus becomes very large and then prohibitive. A synthesis of the TBL excitation focussed on a subdomain of the simulation surface [4] helps reaching higher frequencies while ensuring correct reproduction of the TBL excitation, but limits the observation area to a fraction of the actual panel. Also, some of the proposed methods $[5,6]$ are not able to accurately reproduce the TBL-induced wall-pressure field outside the acoustic wavenumber domain, where the most energetic components of a subsonic TBL are yet located and should be taken into account.

In this context, this study investigates an alternative approach to experimentally predict the vibration response of panels under a TBL excitation by separating the contributions of the forcing wall-pressure excitation from the vibration behavior of the panel. Indeed, the mathematical formulation in the wavenumber domain of a panel vibration response when submitted to random excitations allows estimating the system response at any point on the structure from wall-pressure cross-spectral density (CSD) functions (characterizing the excitation) and from so-called 'sensitivity functions'. The latter are defined as the panel vibration response to wall-pressure acoustic plane waves and characterize the intrinsic vibration behavior of the panel. Since the contributions of the excitation and those from the structural behavior are separated, the method can be fed with numerical and/or experimental data either for the excitation or for the sensitivity functions. This allows performing fast parametric studies by changing the properties of the panel or those of the excitation. 
A method [7] has recently been proposed for estimating the sensitivity functions experimentally without having to excite the panel by sets of surface plane waves which is hard to realize in practice. This alternative approach is based on the reciprocity principle, which states that the sensitivity functions at any point on the structure are equivalent to the panel velocity response expressed in the wavenumber domain when the system is excited by a normal effort at the point of interest. Following this, the experimental process for estimating the sensitivity functions consists in exciting the panel with a transverse force at the point where the panel vibration response is to be determined. The spatial vibratory response of the panel to this force is measured with a scanning laser vibrometer. In a subsequent post-processing phase, a discrete 2-D wavenumber transform of the measured vibratory field normalized by the input force is performed to deduce the sensitivity functions. Finally, by combining the wall-pressure CSD function of the considered random excitation and the previously estimated sensitivity functions, the response of the panel excited by the random excitation can be deduced at the point of interest. This approach has been successfully applied in the case of a panel excited by a diffuse acoustic field (DAF) [7]. In this particular case, the wall-pressure field (WPF) is described in the wavenumber domain by components restricted to the acoustic domain and the sensitivity functions of the panel have to be evaluated only for wavenumbers of magnitude smaller than the acoustic wavenumber.

In principle, the method proposed in [7] for the case of a diffuse acoustic field excitation can be applied for a panel excited by a spatially homogeneous stationary turbulent boundary layer. However, from the authors' knowledge, this has never been assessed experimentally. The work presented in this paper consists in applying and experimentally validating this approach for such an excitation by comparison with direct vibration measurements in an anechoic wind tunnel. This constitutes the main novelty of the paper. Even if the study is limited to the vibration response of a panel to a turbulent boundary layer excitation, its acoustic response (radiated pressure, acoustic intensity) could be further obtained by exciting the panel with a monopole and a dipole source, as pointed out in [7]. In contrast with the DAF, the WPF of a subsonic TBL excitation exhibits components located outside the acoustic domain. The results of the method would therefore be sensitive to the accuracy of the measured sensitivity functions for wavenumbers larger than the acoustic wavenumber. A particular attention is therefore paid to the estimation of these sensitivity functions for a set of wavenumbers adapted for dealing with TBL excitations. Moreover, unlike the DAF 
for which the theoretical CSD functions are well defined [8], various models of the TBL excitation $[9,10]$ exist but none of them is able to accurately describe the WPF induced by a TBL excitation on a large wavenumber range. These models are mostly semi-empirical and can thus be adjusted through parameters like decay rates and convective wavenumber. As there is no clear consensus in the literature regarding universal values for these parameters and/or the model to be used, the WPF of the excitation considered in this study has been measured and used to fit the model of Mellen [11].

The paper is organized as follows: the mathematical formulation of the vibration problem is presented in Sec. II A, and the sensitivity functions involved in the problem are defined in Sec. II B based on the reciprocity principle. The proposed methodology for characterizing the panel vibration response under a TBL is summarized in Sec. II C. The characterization of the WPF under the experimental TBL excitation is presented in Sec. III. In Sec. IV the procedure is validated for an aluminum panel excited by a turbulent flow, based on measured sensitivity functions and an adjusted model of the WPF. The measured sensitivity functions are presented in Sec. IV B. Finally, the complete method is implemented to predict the vibration response of the panel to the experimental TBL, which are compared to direct measurements performed in an anechoic wind tunnel in Sec. IV C.

\section{PROPOSED METHOD FOR ESTIMATING THE VIBRATION RESPONSE OF PANELS UNDER A TURBULENT BOUNDARY LAYER EXCITATIONS}

Let us consider a baffled panel of surface $\Sigma_{p}$ with arbitrary boundary conditions. As illustrated in Fig. 1, a fully developed TBL with a flow velocity $U_{\infty}$ outside the boundary layer is supposed to excite the panel on one of its sides. This excitation is considered stationary in time and spatially homogeneous. The plate and the boundary layer are supposed to be weakly coupled; in other words, the vibration of the plate does not interfere with the WPF and the forcing term is thus not modified by the panel vibration response. This assumption is generally admitted when the panel displacements are much smaller than the characteristic length scales of the flow [12]. The TBL excitation is then characterized by the WPF (i.e., lateral force) induced on a smooth rigid surface. We also define $\mathbf{x}=(x, y)$ an observation point and $\tilde{\mathbf{x}}=(\tilde{x}, \tilde{y})$ an excitation point (where the surface pressure fluctuation induced by the TBL is prescribed). Both points are defined in a Cartesian coordinate system $(x, y, z)$ 
with the origin at the center of the panel, as shown in Fig. 1, and are located on the panel surface $z=0$.

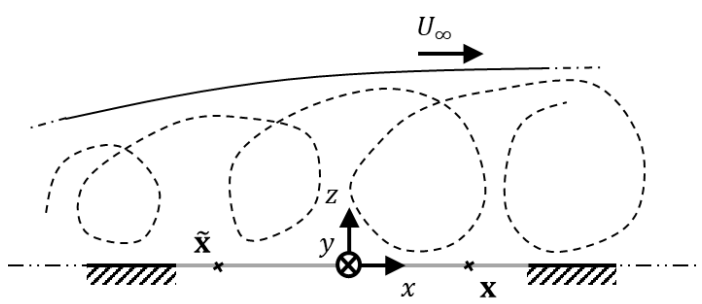

FIG. 1. Illustration of a baffled panel (gray line) excited by a TBL and coordinate system.

To characterize the vibration response of the panel under this excitation, the one-sided normal vibration velocity spectrum $v(\mathbf{x}, f)$ at point $\mathbf{x}$ is considered, where $f$ is the frequency and is considered positive. As the excitation is random, this quantity is derived from the normal velocity auto-spectral density (ASD) function $G_{v v}(\mathbf{x}, f)$. An approach for evaluating this quantity based on deterministic transfer functions and making use of the reciprocity principle has been thoroughly presented in [7] and is briefly summarized in Secs. II A and II B.

\section{A. Mathematical formulation of the vibration response}

The one-sided frequency ASD function of the velocity $G_{v v}(\mathbf{x}, f)$ at point $\mathbf{x}$ can be expressed as the following inverse space-wavenumber Fourier transform:

$$
G_{v v}(\mathbf{x}, f)=\frac{1}{4 \pi^{2}} \iint_{-\infty}^{\infty}\left|H_{v}(\mathbf{x}, \mathbf{k}, f)\right|^{2} G_{p_{b} p_{b}}(\mathbf{k}, f) d \mathbf{k}
$$

where

$$
H_{v}(\mathbf{x}, \mathbf{k}, f)=\iint_{\Sigma_{p}} H_{v / F_{n}}(\mathbf{x}, \tilde{\mathbf{x}}, f) e^{-j \mathbf{k} \tilde{\mathbf{x}}} d \tilde{\mathbf{x}}
$$

where $\mathbf{k}=\left(k_{x}, k_{y}\right)$ is the wavevector defined in the plane $(x, y)$. The function $G_{p_{b} p_{b}}(\mathbf{k}, f)$ corresponds to the CSD function of the WPF on the excitation side (for instance a TBL excitation). The $H_{v}(\mathbf{x}, \mathbf{k}, f)$ function is called the sensitivity function [13] and characterize the vibration behavior of the panel. The term $H_{v / F_{n}}(\mathbf{x}, \tilde{\mathbf{x}}, f)$ corresponds to the transfer function between the panel velocity $v$ at point $\mathbf{x}$ and a normal point force $F_{n}$ applied at point $\tilde{\mathbf{x}}$. According to Eq. (2), the sensitivity function can be interpreted as the vibration 
response of the panel at point $\mathbf{x}$ due to a plane wave excitation with a wavevector $-\mathbf{k}$ (as illustrated in Fig. 2(a)).

Eq. (1) shows that the panel has a filtering effect on the excitation in the wavenumber space [12], which somehow limits the integration to a finite wavenumber domain $\Omega_{\mathbf{k}}$ while ensuring a correct estimation of the integral (see Sec. IV A). This filtering effect allows approximating the integral in Eq. (1) by performing a sum over an appropriately defined finite set of wavevectors $\mathbf{k} \in \Omega_{\mathbf{k}}$ (using the rectangular integration rule). The one-sided frequency ASD function of the velocity at point $\mathbf{x}$ is thereby estimated with

$$
G_{v v}(\mathbf{x}, f) \approx \frac{1}{4 \pi^{2}} \sum_{\mathbf{k} \in \Omega_{\mathbf{k}}}\left|H_{v}(\mathbf{x}, \mathbf{k}, f)\right|^{2} G_{p_{b} p_{b}}(\mathbf{k}, f) \delta \mathbf{k},
$$

where $\delta \mathbf{k}$ represents the wavenumber resolution. To evaluate this quantity, the sensitivity functions $H_{v}$ for wavenumbers belonging to $\Omega_{\mathbf{k}}$ have to be determined.

\section{B. Sensitivity functions based on the reciprocity principle}

In its most general form, the reciprocity principle states that the response of a system is invariant with respect to the exchange of excitation and observation points [14]. For the particular case of a normal force applied at point $\tilde{\mathbf{x}}$ and normal velocity observed at point $\mathbf{x}$, the reciprocity relationship can be translated following the previous notations into [15]

$$
H_{v / F_{n}}(\mathbf{x}, \tilde{\mathbf{x}}, f)=H_{v / F_{n}}(\tilde{\mathbf{x}}, \mathbf{x}, f)
$$

Introducing Eq. (4) in Eq. (2) one obtains

$$
H_{v}(\mathbf{x}, \mathbf{k}, f)=\iint_{\Sigma_{p}} H_{v / F_{n}}(\tilde{\mathbf{x}}, \mathbf{x}, f) e^{-j \mathbf{k} \tilde{\mathbf{x}}} d \tilde{\mathbf{x}}
$$

The right hand side of Eq. (5) can be interpreted as the space-wavenumber transform of $H_{v / F_{n}}(\tilde{\mathbf{x}}, \mathbf{x}, f)$ with respect to the space variable $\tilde{\mathbf{x}}$. The points $\tilde{\mathbf{x}}$ become observation points on the panel surface $\Sigma_{p}$, which means that the space-wavenumber transform is performed over the vibration velocity field of the panel. To sum up, the sensitivity function $H_{v}(\mathbf{x}, \mathbf{k}, f)$ may be obtained by exciting the panel with a normal effort $F_{n}$ at point $\mathbf{x}$ and by calculating the space-wavenumber transform of the transfer function between the panel velocity at the 
observation points and the applied effort (as illustrated in Fig. 2(b)).

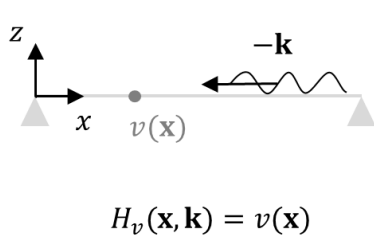

(a)

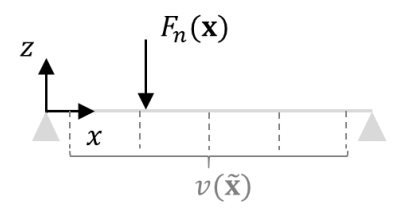

$H_{v}(\mathbf{x}, \mathbf{k})=2 D-D F T[v(\tilde{\mathbf{x}})]$

(b)

FIG. 2. Determination of the sensitivity functions $H_{v}$ : (a) based on the direct interpretation, (b) using the reciprocity principle.

In practice, the vibration field has to be measured on a regular grid of points denoted $\Gamma_{\tilde{\mathbf{x}}}$, using a scanning laser vibrometer for example. The space-wavenumber transform is therefore approximated by a 2D discrete Fourier transform (2D-DFT). In order to avoid aliasing effects, the spatial resolution $\delta \tilde{\mathbf{x}}$ over $\Gamma_{\tilde{\mathbf{x}}}$ should be determined so that the spatial variations of the vibration field can be correctly represented by the grid of points. For a homogeneous isotropic thin panel, $\delta \tilde{\mathbf{x}}$ should be less than or equal to a quarter of the natural flexural wavelength of the panel $\lambda_{f}$ at the highest frequency of interest (as considered in [7]). According to [16], having four points per smallest flexural wavelength allows a nonbiased estimation of the vibration field up to a frequency corresponding to two times this flexural wavenumber. It ensures correct estimation of the sensitivity functions close the flexural wavenumber (where vibratory levels are the largest) and avoids potential aliasing effects. For a more complex panel, a preliminary study should be carried out to define this parameter (for instance, by using a numerical model of the panel or by using a trial and error procedure).

\section{Description of the proposed methodology}

A methodology for experimentally estimating the vibration response of a panel excited by a TBL is derived from Eq. (3) and the sensitivity functions determined using the previously described reciprocity principle. The methodology for evaluating the velocity ASD function $G_{v v}$ at a given point $\mathbf{x}$ of the panel $(z=0)$ can be summarized as follows:

- Excite the panel with a normal mechanical force at point $\mathbf{x}$ and measure the normal velocity response of the panel at points $\tilde{\mathbf{x}} \in \Gamma_{\tilde{\mathbf{x}}}$ to determine $H_{v / F_{n}}(\tilde{\mathbf{x}}, \mathbf{x}, f)$, 
- Perform a 2D-DFT of the panel velocity response $H_{v / F_{n}}(\tilde{\mathbf{x}}, \mathbf{x}, f)$ (with respect to $\tilde{\mathbf{x}}$ ) to obtain the sensitivity functions $H_{v}(\mathbf{x}, \mathbf{k}, f)$ at point $\mathbf{x}$ for $\mathbf{k} \in \Omega_{\mathbf{k}}$,

- Use Eq. (3) and an estimation of the CSD functions of the wall-pressure fluctuations $G_{p_{b} p_{b}}(\mathbf{k}, f)$ to estimate the velocity ASD function $G_{v v}$ at point $\mathbf{x}$ under the considered TBL excitation.

In the following, the vibration behavior of a thin isotropic homogeneous plate will be investigated. The proposed method is however valid for any panel having a linear mechanical behavior and, isotropic and homogeneous conditions are thus not mandatory.

\section{CHARACTERIZATION OF THE EXCITATION}

In addition to the knowledge of the vibration behavior of the panel through the sensitivity functions, solving Eq. (3) requires that CSD functions of the blocked wall-pressure of the excitation are known. Over the past few years, numerous studies have shown that the coherent power of the wall-pressure fluctuations induced by a TBL decays exponentially with the increasing separation distances along flow and transverse directions [17]. It has also been shown that the phase of the cross-spectrum is directly related to the convection wavenumber $k_{c}=\omega / U_{c}$, where $\omega$ is the angular frequency and $U_{c}$ is the convection speed (usually defined as a constant fraction of the free flow velocity $U_{\infty}$ ). These dependencies are included in most of the semi-empirical models $[9,10]$ aiming at predicting the CSD functions of the wall-pressure fluctuations.

In order to validate the proposed methodology in comparison to actual measurements of the panel response in an anechoic wind tunnel, the wall-pressure fluctuations induced by a subsonic turbulent flow generated in a low-speed anechoic wind tunnel have been measured on the considered frequency range in this study $([170,2000 \mathrm{~Hz}]$, see Sec. IV). These measurements will then be used to fit the model of Mellen in Sec. III B.

\section{A. Spiral-shaped surface microphone array}

The wall-pressure fluctuations have been measured at two flow velocities: $U_{\infty}=20 \mathrm{~m} \cdot \mathrm{s}^{-1}$ and $U_{\infty}=40 \mathrm{~m} \cdot \mathrm{s}^{-1}$ using the spiral-shaped rotating microphone array introduced by Robin et al. [18]. 
The array is composed of 61 microphones of three different types in order to reach the desired microphone density and to tackle congestion issues at the center of the array. A deported B\&K 4182 probe and three flush-mounted Knowles FG-23629-P16 microphones are used at the center of the array (as shown in Fig. 3(b)). The remaining part of the array consists of 57 quarter inch pinhole-mounted B\&K 4957 microphones (for further details refer to $[18])$.

The pattern over which the 61 microphones are positioned (red markers in Fig. 3(a)) has been designed so that each microphone has a different radial position with a radial separation of $2 \mathrm{~mm}$. Measurements following 180 consecutive rotations allow reaching an angular resolution of $2^{\circ}$ and thereby reconstructing a high density microphone array at a post-processing step (as illustrated in Fig. 3(a)). Under the assumption of a stationary and homogeneous turbulent WPF, the translation or rotation of an array with at least one reference sensor has proven to be a solution to obtain the needed compromise between a small sensor diameter (to avoid spatial averaging) and small sensor spacing (to gain high spatial resolution) [18] [19]. Since the central microphone position is the only invariant one, it is used as a reference in the calculations of wall-pressure CSD functions.

An amplitude calibration of the microphone array was performed by placing a Larson Davis CAL200 calibrator over each microphone separately and by exciting it with a sine wave of $94 \mathrm{~dB}$ SPL at $1000 \mathrm{~Hz}$. Since the B\&K 4182 probe is deported from the microphone array surface by approximately $4 \mathrm{~cm}$, an additional phase calibration was necessary. To do so, the calibrator was positioned over the three microphones at the center of the array (the B\&K 4182 probe and the two closest Knowles microphones). A simultaneously triggered acoustic pressure signal at $1000 \mathrm{~Hz}$ was extracted for each microphone. The time offset between the signal measured by the deported probe and the signal measured by the two adjacent Knowles microphones (both signals were identical) was compensated in all resulting measurements. Once the signals are expressed in the frequency domain, as a time offset has been applied, the phase calibration was effective regardless of the frequency.

\section{B. Measurement of the wall-pressure fluctuations and adjusted model of Mellen}

The considered TBL-like excitation is reproduced in a low-speed anechoic wind tunnel. The installation consists in a closed-loop wind tunnel powered by two rotating fans. The 


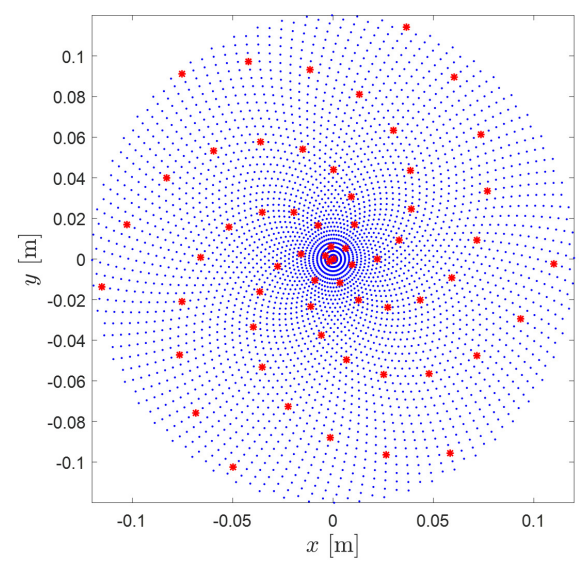

(a)

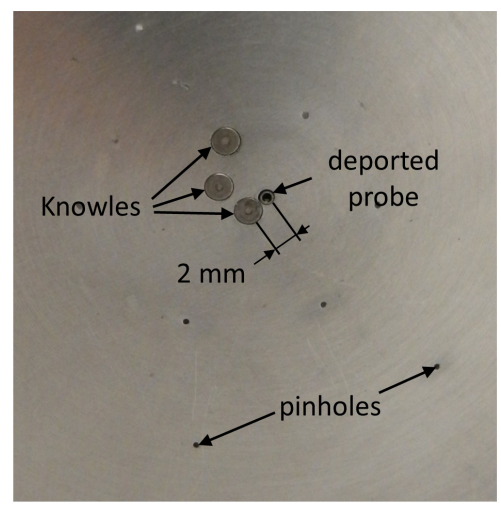

(b)

FIG. 3. (Color online) (a) Microphone positions (red markers) and illustration of a reconstructed grid (blue dots). (b) Close-up view of the mounted sensors and pinholes on measurement side.

air flow is directed to the anechoic chamber through a vent pipe containing a screen to homogenize the flow. A convergent is mounted at the end of the vent pipe inside the anechoic chamber which allows reaching higher flow velocities (up to Mach $\leq 0.12$ ). An $1.22 \times 2.44 \mathrm{~m}^{2}$ plywood panel of $0.019 \mathrm{~m}$ thickness was mounted in the anechoic wind tunnel at the end of the convergent and to help the TBL develop, a sandpaper strip was glued at the end of the convergent (as shown in Fig. 4). The spiral-shaped array was flush-mounted $1.8 \mathrm{~m}$ away from the convergent and 30 seconds acquisitions were performed with a sampling frequency of $8192 \mathrm{~Hz}$. Time signals of the wall-pressure fluctuations were extracted for all microphones and all 180 rotations.

The reconstructed microphone measurement grid theoretically allows reaching a maximum wavenumber of $1570 \mathrm{~m}^{-1}$, which is sufficient to capture the convective contributions over the whole frequency range and for both considered flow velocities (at $2000 \mathrm{~Hz}$ and for 
$U_{\infty}=20 \mathrm{~m} \cdot \mathrm{s}^{-1}$, the convective wavenumber $k_{c}$ equals $838 \mathrm{~m}^{-1}$ with $\left.U_{c}=0.75 \times U_{\infty}\right)$.

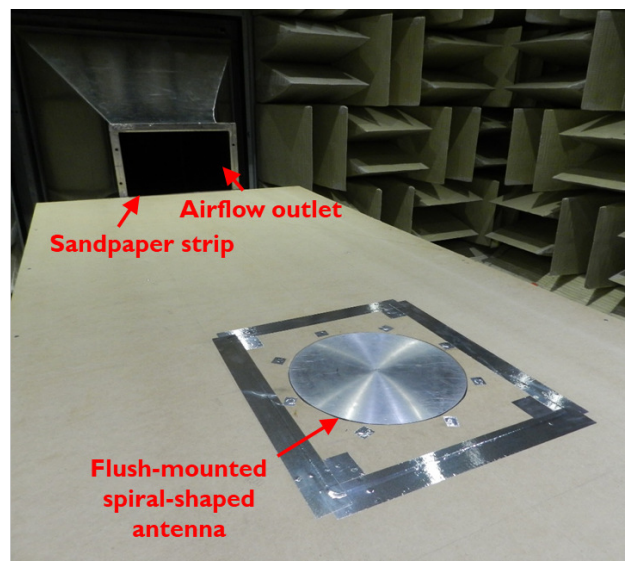

FIG. 4. (Color online) Measurement of the wall-pressure fluctuations using the spiral-shaped surface microphone array flush-mounted in the wind tunnel.

The spatial CSD functions of the wall-pressure fluctuations $G_{p_{b} p_{b}}\left(\xi_{x}, \xi_{y}, f\right)$ were estimated as a function of the spatial separations $\left(\xi_{x}, \xi_{y}\right)$ in both $x$ and $y$ directions using the "cpsd" MATLAB command with a fixed reference point at the center of the array $(x=0, y=0)$. For the remainder of this paper, the "cpsd" MATLAB command was defined with a Hanning window applied to time signals and with a $50 \%$ overlap. Two approaches were investigated to post-process the spatial CSD functions. The first consists in performing a 2D-DFT on $G_{p_{b} p_{b}}\left(\xi_{x}, \xi_{y}, f\right)$ in order to directly estimate the blocked wall-pressure CSD functions in the wavenumber domain [19]. However, the finite dimensions of the microphone array result in unrealistic predictions in the low-wavenumber domain. As the low-wavenumber domain mainly dictates the response of the panel to a TBL excitation (see Sec. IV A), it has to be accurately estimated. Deconvolution methods can be used to compensate for this windowing effect, but they require significant and prohibitive computation time [20].

With this in mind and for computation time to be reasonable, the measurements were fitted to the model of Mellen [11] in the spatial domain

$$
G_{p_{b} p_{b}}\left(\xi_{x}, \xi_{y}, f\right)=G_{p_{b} p_{b}}(f) e^{-\sqrt{\left(\alpha_{x} k_{c} \xi_{x}\right)^{2}+\left(\alpha_{y} k_{c} \xi_{y}\right)^{2}}} e^{j k_{c} \xi_{x}}
$$

where $G_{p_{b} p_{b}}(f)$ is the measured blocked wall-pressure ASD function, $\left(\alpha_{x}, \alpha_{y}\right)$ are the exponential decay rates along $x$ and $y$ directions and $k_{c}$ is the convective wavenumber. Performing a space-wavenumber transform of Eq. (6) yields an expression of blocked wall-pressure CSD functions in the wavenumber domain [11] 


$$
G_{p_{b} p_{b}}(\mathbf{k}, f)=G_{p_{b} p_{b}}(f) \frac{2 \pi\left(\alpha_{x} \alpha_{y} k_{c}^{2}\right)^{2}}{\left[\left(\alpha_{x} \alpha_{y} k_{c}^{2}\right)^{2}+\left(\alpha_{x} k_{c} k_{y}\right)^{2}+\left(\alpha_{y} k_{c}\right)^{2}\left(k_{c}-k_{x}\right)^{2}\right]^{3 / 2}} .
$$

The model of Mellen has been chosen because, like the Corcos model, it can easily be adjusted by estimating $\alpha_{x}, \alpha_{y}$ and $k_{c}$ which is directly related to the convection speed $U_{c}$. Also, the convective peak in the model of Mellen expressed in the wavenumber domain has an oval shape which is in better accordance with the measurements, as opposed to the model of Corcos which has a diamond-like shape [9].

In order to estimate the decay rates $\left(\alpha_{x}, \alpha_{y}\right)$ and convective wavenumber $k_{c}$, the considered model has been fitted to measurements by solving Eq. (6) following the least squares method using the "lsqcurvefit" MATLAB command. First the decay rates have been estimated by solving Eq. (6) while considering the modulus of all the terms in this equation. In a second step, Eq. (6) has been solved by implementing the previously determined decay rates and while considering all terms as complex values to extract $U_{c}$. The extracted parameters are presented in Fig. 5 for the two considered flow velocities.

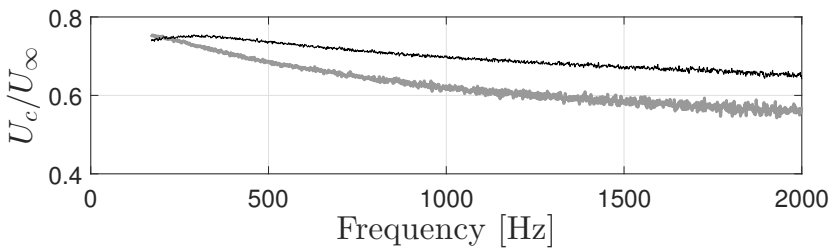

(a)

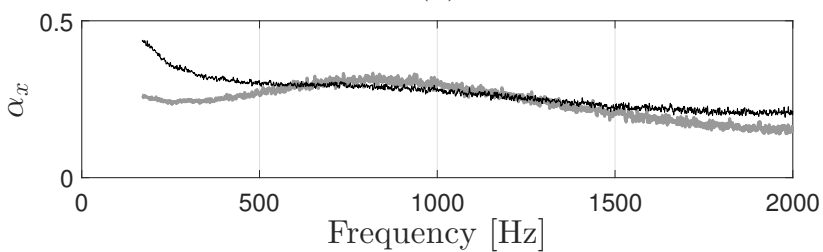

(b)

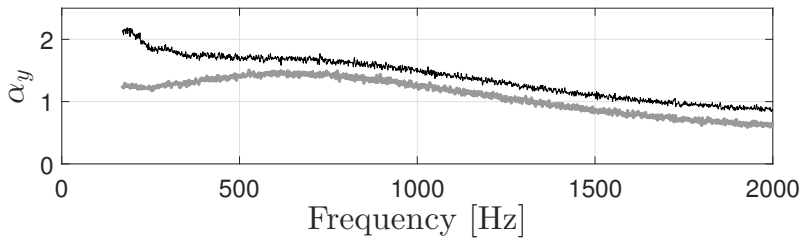

(c)

FIG. 5. TBL parameters extracted from measurements at $U_{\infty}=20 \mathrm{~m} \cdot \mathrm{s}^{-1}$ (bold gray line) and at $U_{\infty}=40 \mathrm{~m} \cdot \mathrm{s}^{-1}$ (light black line) based on the model of Mellen. (a) Convection speed normalized by the flow velocity. (b) Streamwise exponential decay rate $\alpha_{x}$. (c) Spanwise exponential decay rate $\alpha_{y}$. 
The convection speed normalized by the flow velocity is presented in Fig. 5(a). As already observed in the literature $[19,22,23]$, the convection speed normalized by the flow velocity decreases with the increasing frequency and has values in the range [0.55-0.75].

The exponential decay rates estimated along $x$ and $y$ directions are shown in Figs. 5(b) and $5(\mathrm{c})$ respectively. For the two considered flow velocities, the values of decay rates are generally larger than those commonly found in the literature for both Corcos and Mellen models (i.e., $\alpha_{x}=0.116$ and $\alpha_{y}=0.7$ [23]). Like for the convection speed, these estimated decay rates are implemented in the model of Mellen as functions of the frequency.

Finally, the real and imaginary parts of the spatial CSD functions of the blocked wallpressure obtained by fitting the model of Mellen are compared to those derived from measurements with the microphone array in Figs. 6 and 7 for flow velocities of $U_{\infty}=20 \mathrm{~m} \cdot \mathrm{s}^{-1}$ and $U_{\infty}=40 \mathrm{~m} . \mathrm{s}^{-1}$, respectively and at a frequency of $500 \mathrm{~Hz}$.

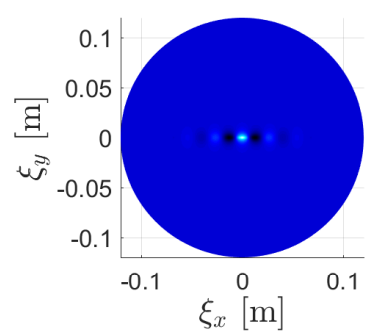

(a)

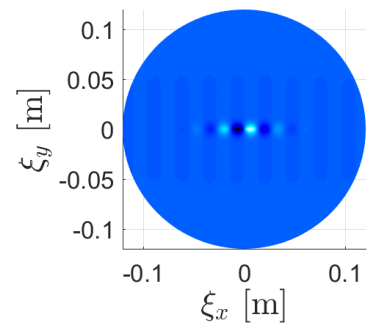

(c)

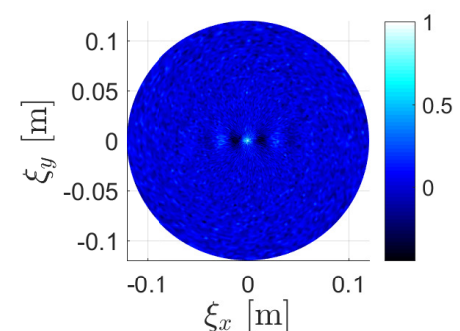

(b)

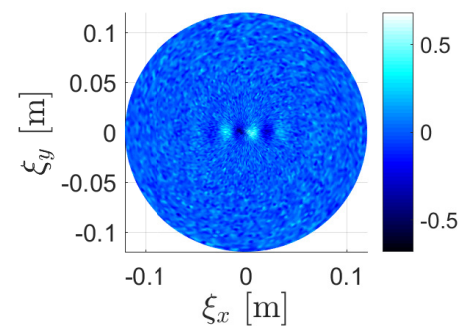

(d)

FIG. 6. (Color online) Spatial CSD function of the blocked wall-pressure normalized by the autospectrum at the center of the array $G_{p_{b} p_{b}}\left(\xi_{x}, \xi_{y}, f\right) / G_{p_{b} p_{b}}(f)$ at $500 \mathrm{~Hz}$ and at a flow velocity $U_{\infty}=20 \mathrm{~m} . \mathrm{s}^{-1}$. (a) Mellen model, real part. (b) Direct measurement, real part. (c) Mellen model, imaginary part. (d) Direct measurement, imaginary part.

Numerically, it has been observed that to correctly estimate the CSD functions of the WPF beneath a TBL, a large number of realizations has to be considered [24]. From an experimental point of view, this can be directly related to the time of acquisition required to average the random process. Since the time required to measure the TBL fluctuations at successive rotated positions of the antenna was already significant (30 seconds per each 


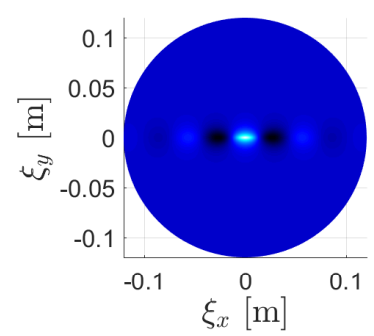

(a)

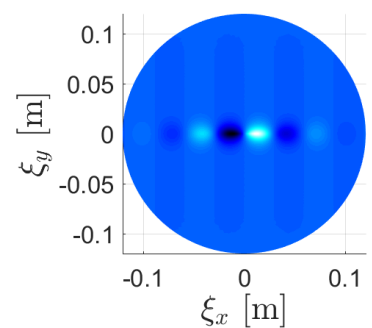

(c)

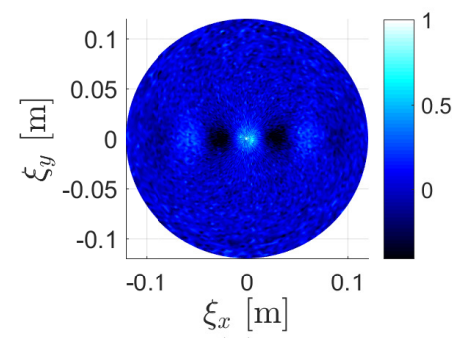

(b)

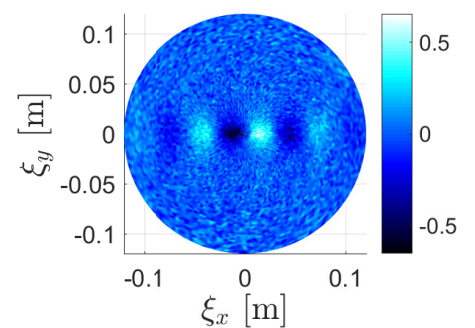

(d)

FIG. 7. (Color online) Spatial CSD function of the blocked wall-pressure normalized by the autospectrum at the center of the array $G_{p_{b} p_{b}}\left(\xi_{x}, \xi_{y}, f\right) / G_{p_{b} p_{b}}(f)$ at $500 \mathrm{~Hz}$ and at a flow velocity $U_{\infty}=40 \mathrm{~m} . \mathrm{s}^{-1}$. (a) Mellen model, real part. (b) Direct measurement, real part. (c) Mellen model, imaginary part. (d) Direct measurement, imaginary part.

consecutive 180 rotations), no attempt was made to increase the acquisition time. However, using a fitted model tends to eliminate measurement noise while ensuring a satisfactory estimation of the wall-pressure fluctuations.

The adjusted model of Mellen is in good agreement with the measurement at both considered flow velocities, which suggests that the proposed method to fit measurements to a model is accurate. It also shows that the extracted parameters can be implemented in the model of Mellen expressed in the wavenumber domain (see Eq. (7)) to apply the proposed methodology for predicting the vibration response of the plate. However, the model of Mellen also needs to define the wall-pressure ASD function $G_{p_{b} p_{b}}(f)$ which is the object of the next paragraph.

Under the assumption of a spatially homogeneous TBL, the auto-spectrum of the wallpressure should be invariant with the observation point. In reality, our measurement results indicate that the auto-spectrum varies (essentially in the streamwise direction). In the proposed approach the wall-pressure auto-spectrum should be estimated at the point of interest $\mathbf{x}$ where the plate response is to be obtained. This information could not be retrieved from this experiment because response point $\mathbf{x}$ considered in Sec. IV was slightly outside and downstream the area covered by the microphone array. The auto-spectra at each micro- 
phones have been estimated using the "cpsd" MATLAB command and their mean value was finally considered for $G_{p_{b} p_{b}}(f)$ in Eq. (3) in the remainder of this work. It is therefore assumed that the spatially averaged ASD function of the blocked wall-pressure $\left\langle G_{p_{b} p_{b}}(f)\right\rangle$ provides an acceptable estimation of the auto-spectrum at all points sufficiently close to the spiral-shaped array. The spatially averaged ASD function of the blocked wall-pressure is presented in Fig. 8 at both considered flow velocities. The trends of the two curves are quite identical. The increase of flow speed results in a nearly constant shift of the auto-spectrum level. The results presented in Figs. 5 and 8 fully describe the parameters used in the model of Mellen which will be considered in Sec. IV C.

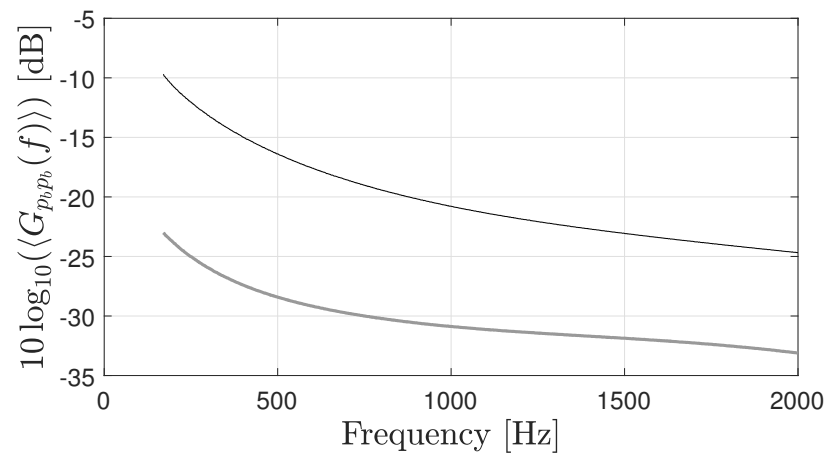

FIG. 8. Spatially averaged ASD function of the blocked wall-pressure $\left\langle G_{p_{b} p_{b}}(f)\right\rangle$ (dB, ref. $1 \mathrm{~Pa}^{2} \cdot \mathrm{Hz}^{-1}$ ) at a flow velocity $U_{\infty}=20 \mathrm{~m} \cdot \mathrm{s}^{-1}$ (bold gray line) and $U_{\infty}=40 \mathrm{~m} \cdot \mathrm{s}^{-1}$ (light black line).

\section{VALIDATION OF THE PROPOSED METHODOLOGY}

\section{A. Test case description}

For experimental validation purposes an academic test case was considered, which consists in a baffled rectangular thin aluminum plate with simply supported boundary conditions on all edges. This baffled plate is submitted to a subsonic TBL air flow on one side. The plate's geometrical and mechanical properties are detailed in Table I. Simply-supported boundary conditions have been chosen because they lead to a simple analytical solution of the plate sensitivity functions. The experimental fabrication method proposed by Robin et al. [21] has been used to setup panels with representative simply supported boundary conditions. 
The considered frequency range is $[170,2000] \mathrm{Hz}$ and the modal structural loss factors $\eta_{m n}$ have been experimentally estimated using the $-3 \mathrm{~dB}$ bandwidth method at all resonance peaks in the considered frequency range. They are taken into account in the numerical simulations and given in Table II with their corresponding resonance frequencies for the first eight resonance peaks.

In this work, the methodology described in Sec. II C was implemented at a given, arbitrary position $\mathbf{x}$ of coordinates $(x=0.18 \mathrm{~m}, y=0.09 \mathrm{~m}, z=0 \mathrm{~m})$ on the plate. Therefore, the quantities of interest are the sensitivity functions $H_{v}$ at point $\mathbf{x}$ and the ASD function of the vibration velocity $G_{v v}(\mathbf{x}, f)$ at point $\mathbf{x}$ when the plate is excited by the TBL.

Like in the previous section, two flow velocities are considered: $U_{\infty}=20 \mathrm{~m} . \mathrm{s}^{-1}$ and $U_{\infty}=40 \mathrm{~m} . \mathrm{s}^{-1}$. The frequency range is well above the aerodynamic coincidence frequency $f_{c}$ defined as the frequency at which the flexural wavenumber $k_{f}$ equals the convective wavenumber $k_{c}$. These two wavenumbers are defined by:

$$
k_{f}=\sqrt{\omega} \sqrt[4]{\frac{\rho h}{D}}
$$

where $D=\frac{E h^{3}}{12\left(1-\nu^{2}\right)}$ is the flexural rigidity and,

$$
k_{c}=\frac{\omega}{U_{c}},
$$

where $U_{c}$ is the convection speed which has been extracted from measurements of the wallpressure fluctuations in Sec. IIIB. The aerodynamic coincidence frequency can thus be expressed as follows

$$
f_{c}=\frac{U_{c}^{2}}{2 \pi} \sqrt{\frac{\rho h}{D}} .
$$

In the considered case, $f_{c}=7.5 \mathrm{~Hz}$ at $U_{\infty}=20 \mathrm{~m} . \mathrm{s}^{-1}$ and $f_{c}=30 \mathrm{~Hz}$ at $U_{\infty}=40 \mathrm{~m} . \mathrm{s}^{-1}$. It is clear from Eq. (1) that the plate filters out the excitation and this filtering effect gets effective above $f_{c}$, in which case $k_{c}>k_{f}$. To illustrate this effect, the theoretical squared absolute value of the sensitivity functions have been plotted in Fig. 9(a) at point $\mathbf{x}$, as a function of $k_{x}\left(k_{y}=0\right)$ and as a function of the frequency (see [7] for details on the numerical model). The strongly decreasing magnitude of $\left|H_{v}\left(\mathbf{x}, k_{x}, f\right)\right|^{2}$ above the flexural wavenumber can be noticed. Similarly, the CSD function of the wall-pressure fluctuations according to the fitted model of Mellen is plotted in Fig. 9(b) while considering the previously obtained decay rates 
and convection speed at a flow velocity of $U_{\infty}=40 \mathrm{~m} \cdot \mathrm{s}^{-1}$. Strong contributions around the convective wavenumber $k_{c}$ can be noticed. Fig. 9(c) shows the product of the squared absolute value of the sensitivity functions and the wall-pressure CSD functions (which is directly involved in the ASD function of the plate velocity), normalized by the maximum value at each frequency. Components are considered filtered out in the $\left(k_{x}, k_{y}\right)$ space when their magnitude is smaller than the maximum value at the corresponding frequency minus $10 \mathrm{~dB}$. In this particular case, the convective wavenumber components centered on $k_{c}$ are entirely filtered out on the whole considered frequency range and the vibration response of the plate to the experimental TBL is mainly driven by the region inside and close to the circle of radius $k_{f}$, the flexural wavenumber. It should be noted that in this work the excitation is a low-speed subsonic TBL. The higher the flow velocity, the lower the slope of the curve $k_{c}(f)$ and therefore, the less the plate filtering effect is effective.

In order to fully characterize the filtering effect of the plate, this study has been performed in both $x$ and $y$ directions and for both considered flow velocities. It allowed retrieving the maximum wavenumbers that need to be considered in Eq. (3) while ensuring a correct estimation of the response of the plate to the experimental TBL. Based on this numerical study and for the considered test case, the wavenumber domain $\Omega_{\mathbf{k}}$ over which Eq. (3) is calculated is limited to wavenumbers $\left|k_{x}\right| \leq 55 \mathrm{~m}^{-1}$ and $\left|k_{y}\right| \leq 55 \mathrm{~m}^{-1}$. These limits are slightly above the flexural wavenumber at $2000 \mathrm{~Hz}\left(k_{f} \approx 51 \mathrm{~m}^{-1}\right)$ which can be explained by the fact that significant contributions remain slightly above the flexural wavenumber (see Fig. 9(c)).

This study on the filtering effect of the plate shows that with an a priori knowledge on the panel, the limits of the wavenumber domain involved in Eqs. (1) and (3) can be minimized while ensuring a correct estimation of the response of the plate to a TBL excitation. From a practical point of view, it allows optimizing the grid of points over which the vibration response of the structure to a normal effort is to be estimated in order to determine the sensitivity functions. For a more complex panel with unknown properties, it might be necessary to extend the wavenumber domain as much as possible (define a refined mesh to determine the sensitivity functions) to minimize errors linked to truncation effects in the wavenumber domain.

To apply the methodology described in Sec. II C, the panel velocity field has to be measured on a grid of points $\Gamma_{\tilde{\mathbf{x}}}$. A uniform mesh of $37 \times 27$ points was considered in directions 


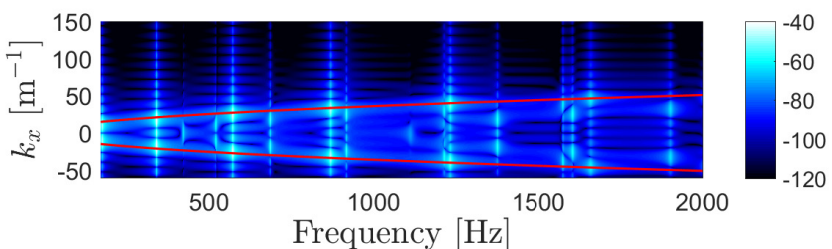

(a)

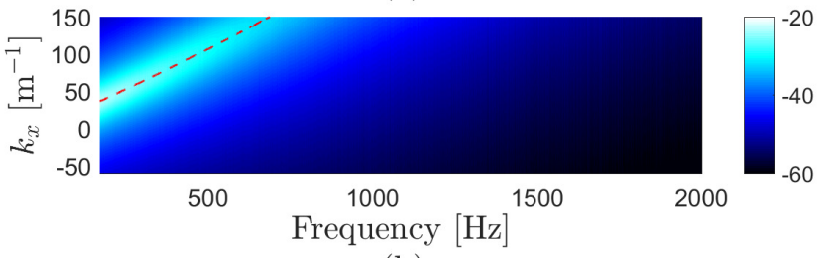

(b)

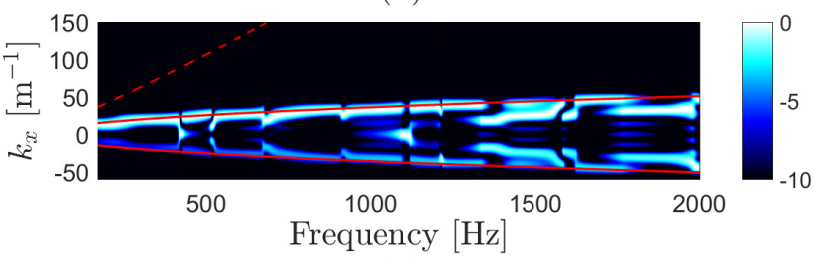

(c)

FIG. 9. (Color online) Illustration of the filtering effect of the plate. (a) Theoretical squared absolute value of the sensitivity functions $\left|H_{v}\left(\mathbf{x}, k_{x}, 0, f\right)\right|^{2}\left(\mathrm{~dB}\right.$, ref. $\left.1 \mathrm{~m} \cdot \mathrm{s}^{-1} \cdot \mathrm{Hz}^{-1}\right)$. (b) Model of Mellen $G_{p_{b} p_{b}}\left(k_{x}, 0, f\right), U_{\infty}=40 \mathrm{~m} \cdot \mathrm{s}^{-1}\left(\mathrm{~dB}\right.$, ref. $\left.1 \mathrm{~Pa}^{2} \cdot \mathrm{Hz}^{-1}\right)$. (c) Product $\left|H_{v}\left(\mathbf{x}, k_{x}, 0, f\right)\right|^{2} \times$ $G_{p_{b} p_{b}}\left(k_{x}, 0, f\right)\left(\mathrm{dB}\right.$, ref. $\left.1 \mathrm{~Pa}^{2} \mathrm{~m}^{2} \cdot \mathrm{s}^{-2} \cdot \mathrm{Hz}^{-1}\right)$ normalized by the maximum value at each frequency. Continuous line: flexural wavenumber $k_{f}$ according to Eq. (8). Dashed line: convective wavenumber $k_{c}$ according to Eq. (9).

$x$ and $y$ respectively and a gap of $1 \mathrm{~cm}$ along the edges was left for practical reasons. This leads to spatial separations of $\delta_{x} \simeq 1.3 \mathrm{~cm}$ and $\delta_{y} \simeq 1.6 \mathrm{~cm}$. These separations are well above the criterion of 4 points per flexural wavelength for all frequencies of interest. The density of points has voluntarily been set to reach wavenumbers higher than the flexural wavenumber in order to experimentally validate the filtering effect of the plate.

Based on this grid of points, the highest wavenumbers $k_{x}^{\max }$ and $k_{y}^{\max }$ that can be resolved in directions $x$ and $y$, respectively, are given by

$$
k_{x}^{\max }=\frac{\pi}{\delta_{x}} \simeq 246 \mathrm{~m}^{-1} ; k_{y}^{\max }=\frac{\pi}{\delta_{y}} \simeq 204 \mathrm{~m}^{-1}
$$

The chosen discretization prevents significant spatial aliasing and thereby ensures a correct estimation of the sensitivity functions on $\Omega_{\mathbf{k}}$. The wavenumber resolutions $\delta k_{x}$ and $\delta k_{y}$ in directions $x$ and $y$ respectively, are given by 


$$
\delta k_{x}=\frac{2 \pi}{L_{x}} \simeq 13 \mathrm{~m}^{-1} ; \delta k_{y}=\frac{2 \pi}{L_{y}} \simeq 15 \mathrm{~m}^{-1}
$$

These wavenumber resolutions are relatively large because of the small dimensions of the panel. In order to improve the wavenumber resolution, zero-padding was used to obtain a wavenumber resolution of $1 \mathrm{~m}^{-1}$ along $k_{x}$ and $k_{y}$.

\section{B. Experimental sensitivity functions}

The accuracy of the reciprocity approach for evaluating the panel sensitivity functions has been assessed in [7] for wavenumbers restricted to the acoustic wavenumber circle (of radius defined by $k_{0}=\omega / c_{0}$, with $c_{0}$ the speed of sound). In this work, the sensitivity functions have to be determined on a larger wavenumber domain (inside and close to the flexural wavenumber circle of radius $k_{f}$ ).

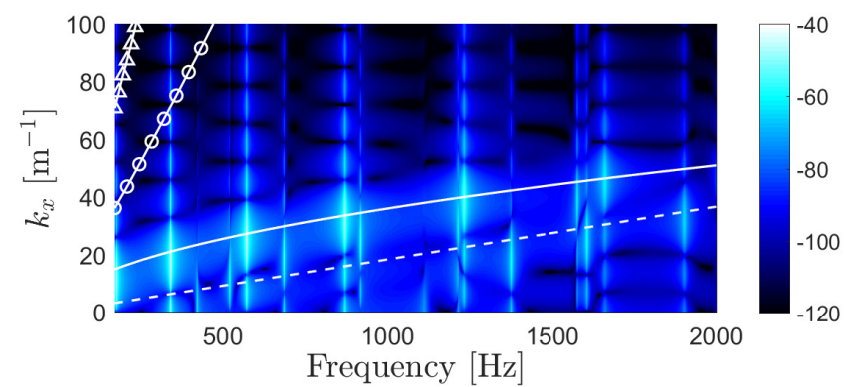

(a)

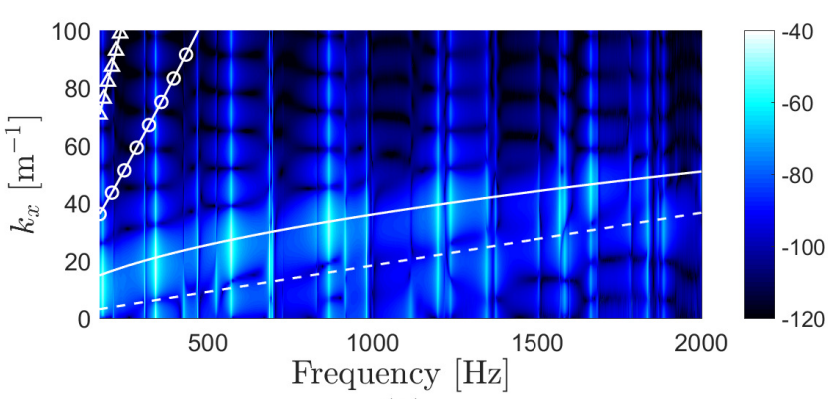

(b)

FIG. 10. (Color online) Squared absolute value of the sensitivity functions $\left|H_{v}(\mathbf{x}, \mathbf{k}, f)\right|^{2}$ (dB, ref. $1 \mathrm{~m}^{2} \cdot \mathrm{s}^{-2} \cdot \mathrm{Hz}^{-1}$ ) along $k_{x} \geqslant 0$ for $k_{y}=0$ : (a) numerical result, (b) experimental result. The superimposed lines represent: $k_{f}$ according to Eq. (8) (continuous line); $k_{0}$ (dashed line); $k_{c}$ according to Eq. (9) for $U_{\infty}=20 \mathrm{~m} . \mathrm{s}^{-1}$ (line with triangle markers); $k_{c}$ according to Eq. (9) for $U_{\infty}=40 \mathrm{~m} \cdot \mathrm{s}^{-1}$ (line with circle markers). 
The sensitivity functions of the panel have been estimated from measurements based on the methodology described in Sec. IIC. A normal effort was applied at point $\mathbf{x}$ using a TMS SmartShaker K2007E01 with integrated amplifier, which was fed with a swept sine over the considered frequency range and the force was measured using an impedance head PCB288D01. An adapter was used between the impedance head and the plate reducing the area of mechanical coupling to approximately a $5 \mathrm{~mm}$ diameter circle. The vibratory response of the panel was measured on the grid of $37 \times 27$ points with a single point scanning laser vibrometer (PSV-300 Polytec) and a time Fourier transform was directly performed in the post-processing software with ten linear averages. A frequency resolution of $0.625 \mathrm{~Hz}$ was chosen and fixed for the remainder of this paper. The numerically obtained sensitivity functions in Fig. 10(a) (see [7] for details on the numerical model) are compared to the experimental sensitivity functions in Fig. 10(b). In both cases, the sensitivity functions are presented along $k_{x} \geqslant 0$ and for $k_{y}=0$ as a function of the frequency. The panel vibration modes are noticeable below or close to the dispersion curve of the flexural motion, Eq. (8). Since the plate is considered isotropic, its vibration behavior is similar in both direction which explains why results are only presented in the flow-direction, where the excitation has its most energetic components. A representation along $k_{y}$ would have shown similar results with differences linked to the dimensions of the plate resulting in different modal wavenumbers in direction $y$. Results presented in Fig. 10 show that the experimentally obtained sensitivity functions are in good agreement with numerical simulations on the whole frequency range. Slight differences between experimental and numerical results can be observed mainly for the upper part of the frequency range. They can be attributed to the boundary conditions of the actual plate, which are close to simply supported boundary conditions but not absolutely perfect.

\section{Application of the proposed methodology and comparison with direct mea- surements in the wind tunnel}

The vibration response of the plate estimated from the proposed approach (Eq. (3)) is finally compared to direct measurements in the wind tunnel. Measurements in the wind tunnel were performed with a plate identical to the one used for sensitivity functions measurement (Sec. IV B) and described in Table I (similar dimensions, material and boundary 
conditions). The plate was flush-mounted at the location of the previously mounted spiralshaped array (as shown in Fig. 11). Aside from replacing the spiral-shaped array with the plate, the experimental conditions remained unchanged in order to keep the turbulent flow excitation identical to the one characterized in Sec. III.

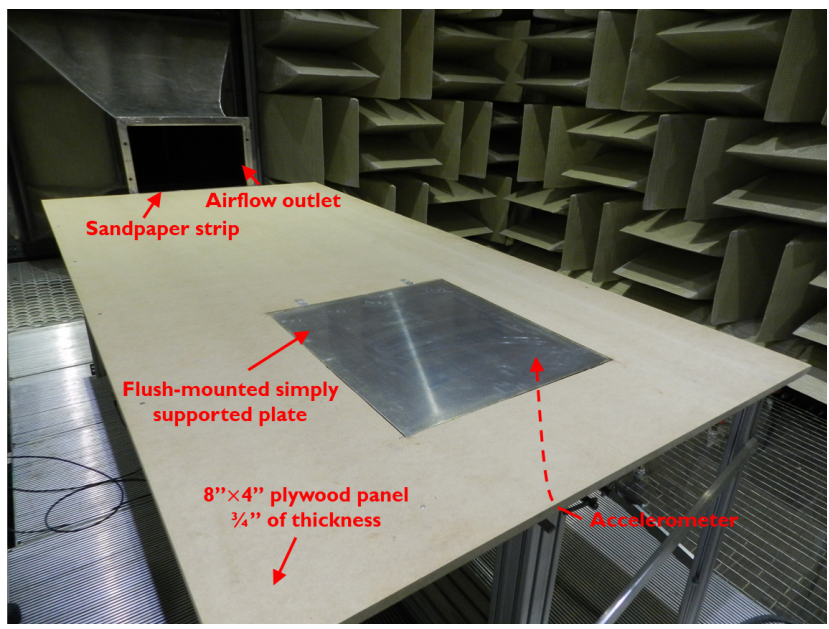

FIG. 11. (Color online) Experimental setup used to measure the vibration response of the plate to the TBL excitation generated in the wind tunnel.

On the one hand, the vibration velocity of the plate was measured at point $\mathbf{x}$ using a PCB 353B18 accelerometer, the acceleration signal over time was extracted from the postprocessing software with a sampling frequency of $8192 \mathrm{~Hz}$ and the vibration velocity ASD function $G_{v v}$ was estimated using the "cpsd" MATLAB command. On the other hand, the vibration velocity ASD function was estimated by applying the proposed methodology using Eq. (3), the measured sensitivity functions $H_{v}(\mathbf{x}, \mathbf{k}, f)$ and the fitted Mellen model of the WPF CSD function $G_{p_{b} p_{b}}(\boldsymbol{k}, f)$.

The plate velocity ASD function measured in the anechoic wind tunnel room at point $\mathbf{x}$ is compared to the result obtained with the proposed method in Figs. 12 and 13 for flow velocities of $20 \mathrm{~m} \cdot \mathrm{s}^{-1}$ and $40 \mathrm{~m} \cdot \mathrm{s}^{-1}$, respectively. One can notice that all modes are excited by the reproduced TBL. According to the formulation presented in Eq. (1), where both functions in the integral are positive or null, any type of mode (even or odd) can be excited by any random excitation.

For both considered flow velocities, slight shifts of the resonance peaks in the high frequency range are noticed. They can be explained by the fact that an adapter was used between the impedance head and the plate for the measurement of the sensitivity functions, 


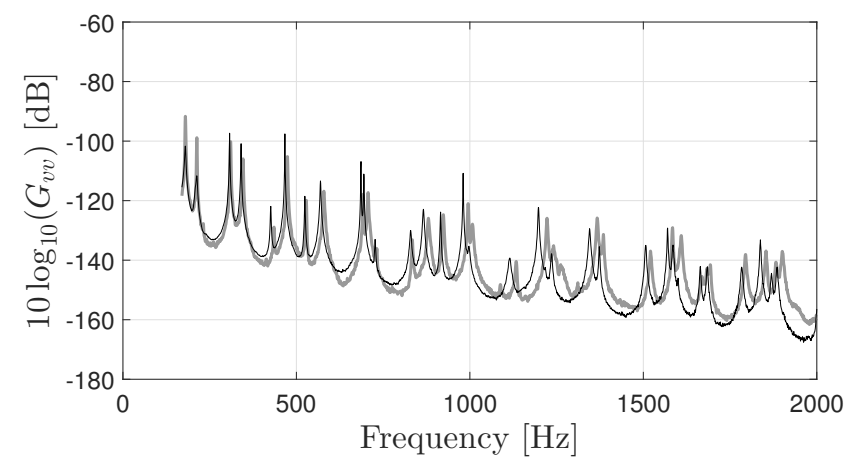

FIG. 12. Velocity ASD functions $G_{v v}\left(\mathrm{~dB}\right.$, ref. $\left.1 \mathrm{~m}^{2} \cdot \mathrm{s}^{-2} \cdot \mathrm{Hz}^{-1}\right)$ at flow velocity $U_{\infty}=20 \mathrm{~m} \cdot \mathrm{s}^{-1}$ : proposed approach (light black line) vs. wind tunnel measurements (bold gray line).

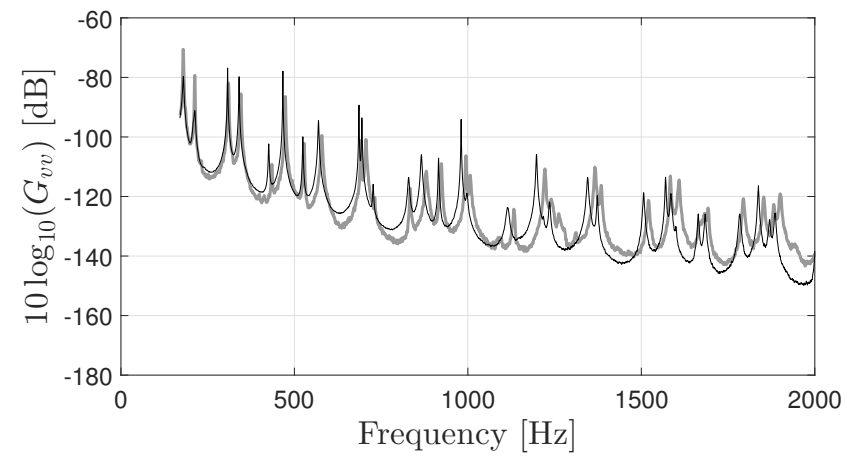

FIG. 13. Velocity ASD functions $G_{v v}\left(\mathrm{~dB}\right.$, ref. $\left.1 \mathrm{~m}^{2} \cdot \mathrm{s}^{-2} \cdot \mathrm{Hz}^{-1}\right)$ at flow velocity $U_{\infty}=40 \mathrm{~m} \cdot \mathrm{s}^{-1}$ : proposed approach (light black line) vs. wind tunnel measurements (bold gray line).

adding a mass to the system. It can also be explained by the fact that the sensitivity functions were not measured on the same plate as the plate installed in the wind tunnel to directly measure the response. Despite all efforts made to have two identical panels, slight differences in dimensions, material properties and boundary conditions were unavoidable. The structural damping is implicitly taken into account through the measurement of the sensitivity functions in the proposed approach and through the direct measurement of the vibration response in the wind tunnel. The differences in peak values, as well as off resonance values, can again be linked to the fact that the two plates had differences in structural damping values. For instance, the damping loss factors estimated at the first two peaks for the plate mounted in the wind tunnel $\left(\eta_{21}=0.55 \%\right.$ and $\left.\eta_{12}=0.56 \%\right)$ are lower than those estimated on the plate used to measure the sensitivity functions (given in Table II). For the following six peaks, an opposite trend has been observed which is in line with obtained 
results and, thereby, explains the amplitude differences at the resonance peaks.

The differences can also be attributed to the fact that the auto-spectrum of the blocked wall-pressure has not been estimated directly at point $\mathbf{x}$ but by averaging the auto-spectra over the area covered by the spiral-shaped array. This leads to slight errors in terms of overall trend and thereby also contributes to the under-estimation by the proposed method, particularly at high frequencies. Despite the differences at the first two peaks of resonance, which again are linked to discrepancies in the structural loss factors of both plates, the predicted and measured data are in good agreement, which shows that for the considered test case, the velocity response of the plate can be fairly well estimated experimentally by applying the proposed methodology.

\section{CONCLUSION}

In this paper, an alternative methodology for characterizing the vibration response of panels to a turbulent boundary layer excitation was proposed. This approach is based on the concept that the panel response at a point $\mathbf{x}$ on the panel to a random pressure field depends on two quantities in the wavenumber domain. First, the wall-pressure cross-spectral density function, which characterizes the excitation. Second, so-called 'sensitivity functions' determined at point $\mathbf{x}$ and which characterize the dynamic behavior of the panel. Those sensitivity functions can be determined using the reciprocity principle, which states that they are equivalent to the panel velocity frequency response function when the panel is excited by a normal force at the point of interest $\mathbf{x}$, expressed in the wavenumber domain. The sensitivity functions can be estimated easily by experiments based on this reciprocal interpretation. The method has been validated experimentally for a simply supported aluminum plate. The confrontation to direct measurements in an anechoic wind tunnel has shown that a fairly good estimate of the vibration response can be obtained by applying the proposed methodology. This indicates that the excitation, as well as the panel behavior, have been correctly characterized.

The main limitations of the proposed approach rely on the assumptions of the mathematical formulation of the problem: the system should be linear (i.e., elastic material, small deformations) and time invariant. It is also assumed that the wall-pressure field exciting the panel corresponds to that of the turbulent flow in rigid conditions. Models provided in the 
literature can be used but should be adjusted to actual measurements in order to correctly represent the considered excitation (it is however not mandatory to apply the method).

From a practical point of view, the acquisition time for the reciprocal approach is significantly longer than for a direct measurement in the wind tunnel because a scanning laser vibrometer was used. However, it could be well reduced with the recently developed fullfield vibration measuring techniques (such as digital image correlation or deflectometry [25]). Once the excitation is characterized, the overall cost for a measurement in a given facility as well as variability between measurements in different ones can be greatly reduced using the proposed approach.

The strongest asset of the proposed methodology is that it allows performing an ex situ characterization of a panel under turbulent boundary layer excitation. The characterization of the excitation and/or the panel can be experimental, but might as well come from numerical models. This approach is therefore well suited for parametric studies. Once the excitation is defined, the response of panels with various mechanical properties under the considered excitation can easily be deduced.

\section{ACKNOWLEDGMENTS}

This work was supported by the Labex CeLyA of Université de Lyon, operated by the French National Research Agency (ANR-10-LABX-0060/ANR-11-IDEX-0007). Special thanks must go to Mr. Patrick Lévesque for his considerable contribution to all experimental setups required to perform this study.

[1] M. Aucejo, L. Maxit, J.-L. Guyader, "Experimental simulation of turbulent boundary layer induced vibrations by using a synthetic array", J. Sound Vib. 331(16), 3824-3843 (2012). doi:10.1016/j.jsv.2012.04.010

[2] T. Bravo, C. Maury, "The experimental synthesis of random pressure fields: Methodology", J. Acoust. Soc. Am. 120(5), 2702-2711 (2006). doi:10.1121/1.2354008

[3] T. Bravo, C. Maury, "A synthesis approach for reproducing the response of aircraft panels to a turbulent boundary layer excitation", J. Acoust. Soc. Am. 129(1), 143-153 (2011). doi : $10.1121 / 1.3514530$ 
[4] C. Maury, T. Bravo, "Focussed Synthesis of a Turbulent Boundary Layer Excitation", 22nd AIAA/CEAS Aeroacoustics Conference, Aeroacoustics Conferences, pp. 1-12 (2013). doi: $10.2514 / 6.2016-2763$

[5] A. Berry, R. Dia, O. Robin, "A wave field synthesis approach to reproduction of spatially correlated sound fields", J. Acoust. Soc. Am. 131(2), 1226-1239 (2012). doi:10.1121/1. 3675942

[6] O. Robin, A. Berry, S. Moreau, "Experimental vibroacoustic testing of plane panels using synthesized random pressure fields", J. Acoust. Soc. Am. 135(6), 3434-3445 (2014). doi: $10.1121 / 1.4872298$

[7] C. Marchetto, L. Maxit, O. Robin, A. Berry, "Vibroacoustic response of panels under diffuse acoustic field excitation from sensitivity functions and reciprocity principles", J. Acoust. Soc. Am. 141(6), 4508-4521 (2017). doi:10.1121/1.4985126

[8] B. Rafaely, "Spatial-temporal correlation of a diffuse sound field", J. Acoust. Soc. Am. 107(6), 3254-3258 (2000). doi:10.1121/1.429397

[9] T. S. Miller, J. M. Gallman, and M. J. Moeller, "Review of Turbulent Boundary Layer Models for Acoustic Analysis", 49th AIAA Aerospace Sciences Meeting, pp. 1-20 (2011). doi:10. 2514/1. C031405

[10] A. Caiazzo, R. D'Amico, W. Desmet, "A Generalized Corcos model for modelling turbulent boundary layer wall pressure fluctuations", J. Sound Vib. 372, 192-210 (2016). doi : 10.1016/ j.jsv.2016.02.036

[11] R. H. Mellen, "Wave-vector filter analysis of turbulent flow", J. Acoust. Soc. Am. 95(3), 3885-3899 (1994). doi:10.1121/1.408556

[12] C. Maury, P. Gardonio, S. J. Elliott, "A wavenumber approach to modelling the response of a randomly excited panel, part 1: general theory", J. Sound Vib. 252(1), 83-113 (2002). doi:10.1006/jsvi.2001.4028

[13] Y. K. Lin, "Probabilistic theory of structural dynamics", p. 207, McGraw-Hill, New York (1967).

[14] F. J. Fahy, "Some Applications of the Reciprocity Principle in Experimental Vibroacoustics", Acoustical Physics 49(2), 217-229 (2003). doi:10.1134/1.1560385

[15] L. Maxit, V. Denis, "Prediction of flow induced sound and vibration of periodically stiffened plates", J. Acoust. Soc. Am. 133(1), 146-160 (2013). doi:10.1121/1.4768875 
[16] M. Unser, "Sampling - 50 years after Shannon", Proc. IEEE 88(4), 569-587 (2000). doi: $10.1109 / 5.843002$

[17] D. Palumbo, "Determining correlation and coherence lengths in turbulent boundary layer flight data", J. Sound Vib. 331, 3721-3737 (2012). doi:10.1016/j.jsv.2012.03.015

[18] O. Robin, S. Moreau, T. Padois, A. Berry, "Measurement of the wavenumber-frequency spectrum of wall pressure fluctuations: spiral-shaped rotative arrays with pinhole-mounted quarter inch microphones", 19th AIAA/CEAS Aeroacoustics Conference, Aeroacoustics Conferences, pp. 1-18 (2013). doi:10.2514/6.2013-2058

[19] B. Arguillat, D. Ricot, C. Bailly, G. Robert, "Measured wavenumber: Frequency spectrum associated with acoustic and aerodynamic wall pressure fluctuations", J. Acoust. Soc. Am. 128(4), 1647-1655 (2010). doi:10.1121/1.3478780

[20] K. Ehrenfried, L. Koop, "Experimental study of pressure fluctuations beneath a compressible turbulent boundary layer", 14th AIAA/CEAS Aeroacoustics Conference, Aeroacoustics Conferences, pp. 1-18 (2008). doi:10.2514/6.2008-2800

[21] O. Robin, J-D. Chazot, R. Boulandet, M. Michau, A. Berry, N. Atalla, "A plane and thin panel with representative simply supported boundary conditions for laboratory vibroacoustic test", Acta Acust. United Ac. 102(1), 170-182 (2016). doi:10.3813/AAA.918934

[22] H. H. Schloemer, "Effects of Pressure Gradients on Turbulent-Boundary-Layer Wall-Pressure Fluctuations", J. Acoust. Soc. Am. 42(1), 93-113 (1967). doi:10.1121/1.1910581

[23] N. Totaro, G. Robert, J. L. Guyader, "Frequency Averaged Injected Power under Boundary Layer Excitation: An Experimental Validation", Acta Acust. United Ac. 94(4), 534-547 (2008). doi:10.3813/AAA. 918062

[24] L. Maxit, "Simulation of the pressure field beneath a turbulent boundary layer using realizations of uncorrelated wall plane waves", J. Acoust. Soc. Am. 140(2), 1268-1285 (2016). doi:10.1121/1.4960516

[25] M. Grédiac, F. Hild, "Full-Field Measurements and Identification in Solid Mechanics", Chap. 3 and 6, ISTE Ltd. and John Wiley \& Sons Inc., London (2013). 
Fig. 1. Illustration of a baffled panel (gray line) excited by a TBL and coordinate system.

Fig. 2. Determination of the sensitivity functions $H_{v}$ : (a) based on the direct interpretation, (b) using the reciprocity principle.

Fig. 3. (a) Microphone positions (red markers) and illustration of a reconstructed grid (blue dots). (b) Close-up view of the mounted sensors and pinholes on measurement side.

Fig. 4. Measurement of the wall-pressure fluctuations using the spiral-shaped surface microphone array flush-mounted in the wind tunnel.

Fig. 5. TBL parameters extracted from measurements at $U_{\infty}=20 \mathrm{~m} \cdot \mathrm{s}^{-1}$ (bold gray line) and at $U_{\infty}=40 \mathrm{~m} \cdot \mathrm{s}^{-1}$ (light black line) based on the model of Mellen. (a) Convection speed normalized by the flow velocity. (b) Streamwise exponential decay rate $\alpha_{x}$. (c) Spanwise exponential decay rate $\alpha_{y}$.

Fig. 6. Spatial CSD function of the blocked wall-pressure normalized by the autospectrum at the center of the array $G_{p_{b} p_{b}}\left(\xi_{x}, \xi_{y}, f\right) / G_{p_{b} p_{b}}(f)$ at $500 \mathrm{~Hz}$ and at a flow velocity $U_{\infty}=20 \mathrm{~m} \cdot \mathrm{s}^{-1}$. (a) Mellen model, real part. (b) Direct measurement, real part. (c) Mellen model, imaginary part. (d) Direct measurement, imaginary part.

Fig. 7. Spatial CSD function of the blocked wall-pressure normalized by the autospectrum at the center of the array $G_{p_{b} p_{b}}\left(\xi_{x}, \xi_{y}, f\right) / G_{p_{b} p_{b}}(f)$ at $500 \mathrm{~Hz}$ and at a flow velocity $U_{\infty}=40 \mathrm{~m} \cdot \mathrm{s}^{-1}$. (a) Mellen model, real part. (b) Direct measurement, real part. (c) Mellen model, imaginary part. (d) Direct measurement, imaginary part.

Fig. 8. Spatially averaged ASD function of the blocked wall-pressure $\left\langle G_{p_{b} p_{b}}(f)\right\rangle(\mathrm{dB}$, ref. $4 \times 10^{-10} \mathrm{~Pa}^{2} \cdot \mathrm{Hz}^{-1}$ ) at a flow velocity $U_{\infty}=20 \mathrm{~m} \cdot \mathrm{s}^{-1}$ (bold gray line) and $U_{\infty}=40 \mathrm{~m} \cdot \mathrm{s}^{-1}$ (light black line).

Fig. 9. Illustration of the filtering effect of the plate. (a) Theoretical squared absolute value of the sensitivity functions $\left|H_{v}\left(\mathbf{x}, k_{x}, 0, f\right)\right|^{2}\left(\mathrm{~dB}\right.$, ref. $\left.1 \mathrm{~m} \cdot \mathrm{s}^{-1} \cdot \mathrm{Hz}^{-1}\right)$. Model of Mellen $G_{p_{b} p_{b}}\left(k_{x}, 0, f\right), U_{\infty}=40 \mathrm{~m} \cdot \mathrm{s}^{-1}$ (dB, ref. $\left.1 \mathrm{~Pa}^{2} \cdot \mathrm{Hz}^{-1}\right)$. (c) Product 

$\left|H_{v}\left(\mathbf{x}, k_{x}, 0, f\right)\right|^{2} \times G_{p_{b} p_{b}}\left(k_{x}, 0, f\right)\left(\mathrm{dB}\right.$, ref. $\left.1 \mathrm{~Pa}^{2} \mathrm{~m}^{2} \cdot \mathrm{s}^{-2} \cdot \mathrm{Hz}^{-1}\right)$ normalized by the maximum value at each frequency. Continuous line: flexural wavenumber $k_{f}$ according to Eq. (8). Dashed line: convective wavenumber $k_{c}$ according to Eq. (9).

Fig. 10. Squared absolute value of the sensitivity functions $\left|H_{v}(\mathbf{x}, \mathbf{k}, f)\right|^{2}$ (dB, ref. $1 \mathrm{~m}^{2} \cdot \mathrm{s}^{-2} \cdot \mathrm{Hz}^{-1}$ ) along $k_{x} \geqslant 0$ for $k_{y}=0$ : (a) numerical result, (b) experimental result. The superimposed lines represent: $k_{f}$ according to Eq. (8) (continuous line); $k_{0}$ (dashed line); $k_{c}$ according to Eq. (9) for $U_{\infty}=20 \mathrm{~m} \cdot \mathrm{s}^{-1}$ (line with triangle markers); $k_{c}$ according to Eq. (9) for $U_{\infty}=40 \mathrm{~m} \cdot \mathrm{s}^{-1}$ (line with circle markers).

Fig. 11. Experimental setup used to measure the vibration response of the plate to the TBL excitation generated in the wind tunnel.

Fig. 12. Velocity ASD functions $G_{v v}\left(\mathrm{~dB}\right.$, ref. $\left.1 \mathrm{~m}^{2} \cdot \mathrm{s}^{-2} \cdot \mathrm{Hz}^{-1}\right)$ at flow velocity $U_{\infty}=$ $20 \mathrm{~m} . \mathrm{s}^{-1}$ : proposed approach (light black line) vs. wind tunnel measurements (bold gray line).

Fig. 13. Velocity ASD functions $G_{v v}\left(\mathrm{~dB}\right.$, ref. $\left.1 \mathrm{~m}^{2} \cdot \mathrm{s}^{-2} \cdot \mathrm{Hz}^{-1}\right)$ at flow velocity $U_{\infty}=$ $40 \mathrm{~m} . \mathrm{s}^{-1}$ : proposed approach (light black line) vs. wind tunnel measurements (bold gray line). 
TABLE I. Mechanical properties of the simply supported aluminum plate.

\begin{tabular}{cc}
\hline \hline Parameter (Symbol), Unit & Value \\
\hline Young's modulus $(E), \mathrm{GPa}$ & 68.9 \\
Poisson's ratio $(\nu)$ & 0.3 \\
Mass density $(\rho), \mathrm{kg} / \mathrm{m}^{3}$ & 2740 \\
Length $\left(L_{x}\right), \mathrm{mm}$ & 480 \\
Width $\left(L_{y}\right), \mathrm{mm}$ & 420 \\
Thickness $(h), \mathrm{mm}$ & 3.17 \\
\hline \hline
\end{tabular}

TABLE II. Modal properties of the simply supported aluminum plate.

\begin{tabular}{ccc}
\hline \hline $\boldsymbol{( m , \boldsymbol { n } )}$ & Resonance frequency, $\boldsymbol{f}_{\boldsymbol{m} \boldsymbol{n}}[\mathrm{Hz}]$ & Modal structural loss factor, $\boldsymbol{\eta}_{\boldsymbol{m} \boldsymbol{n}}[\%]$ \\
\hline$(2,1)$ & 180 & 1.71 \\
$(1,2)$ & 213.1 & 2.05 \\
$(2,2)$ & 307.5 & 0.28 \\
$(3,1)$ & 340.6 & 0.3 \\
$(1,3)$ & 426.3 & 0.5 \\
$(3,2)$ & 466.9 & 0.11 \\
$(2,3)$ & 524.4 & 0.29 \\
$(4,1)$ & 569.4 & 0.41 \\
\hline \hline
\end{tabular}

